\title{
Screening Acetylcholinesterase Inhibitors from Marine-Derived Actinomycetes by Simple Chromatography
}

\author{
Mudyawati Kamaruddin $^{1^{*}}$, Ismail Marzuki ${ }^{2}$, Rasheed Ahmad ${ }^{3^{*}}$, Asril Burhan ${ }^{4}$ \\ ${ }^{1}$ Midwifery Academy of Tahirah Al Baeti Bulukumba, Bulukumba, Indonesia \\ ${ }^{2}$ Department of Chemical Engineering, Fajar University, Makassar, Indonesia \\ ${ }^{3}$ Faculty of Science and Technology, Airlangga University, Surabaya, Indonesia \\ ${ }^{4}$ Sekolah Tinggi Ilmu Farmasi Makassar, Indonesia \\ *E-mail: mudya07@gmail.com.; rasheed.ahmad@,fst.unair.ac.id
}

\begin{abstract}
Acetylcholinesterase (AChE) is an enzyme involved in the metabolic hydrolysis of Acetylcholine (Ach) cholinergic synapses in the central and peripheral nervous systems. As a consequence, $\mathrm{AChE}$ inhibitors facilitate an increase in the concentration and duration of Ach synaptic activity. Actualy, acetylcholinesterase inhibitors (AChEIs) are also the best therapeutic agents effective for Alzheimer's patients. The potential sources of the current AChE inhibitors are mainly plants. However, some of these medications are considered to have limitations such as a short half-life or side-effect such as hepatotoxicity. Marine microorganisms are a possible new source of the enzyme inhibitors. Since their rich and diverse metabolites have a broad range of biological activities, one of them is actinomycetes of special interest in antibiotics. The aim of this study was to screen for AChE inhibitors from marine-derived actinomycetes by a simple method, thin layer chromatography (TLC). Furthermore, the TLC used to determination of solvents for adsorption of AchE inhibitors from actinomycetes. The results showed that the combination of chloroform $/$ methanol $=80 / 20$ gave the best AchE inhibitors separation results, while the combination of DCM $/$ methanol $=90 / 10$ should be considered if gradient elution was selected in the separation process.
\end{abstract}

Keywords: Acetylcholinesterase, AChE Inhibitor, Thin Layer Chromatography 


\section{Introduction}

For thousands of years, natural products have played a significant role in the treatment and prevention of human diseases worldwide. Natural medicinal items have been extracted from a range of sources including terrestrial plants and microorganisms, marine organisms (invertebrates, plants and microorganisms) (Chin Y-W, et al. 2006). Marine bacteria may produce chemical compounds as bioactive natural products, which may be derived from the primary or, rather, secondary metabolism of these species. Among such marine bacterial, gram-positive actinomycetes bacteria predominate in soil and are of particular interest, as they are known to generate chemically diverse compounds with a wide range of biological activities, and are of high pharmacological and commercial interest. Actinomycetes are best known for their ability to produce antibiotics that are not limited to antibacterial antibiotics that include antifungal, anticancer, and antiviral antibiotics (Arifuzzaman et al, 2010).

Because of their ability for producing antibiotics, more than 12,000 antibiotics have been discovered in the last 55 years of which actinomycetes constitutes $70 \%$ and $30 \%$ is by fungi and other microorganisms (Nanjwade et al., 2010). However, the ability of actinomycetes to produce different enzymes is given less concern which is the subject of our research. Our Laboratory has obtained and characterized actinomycetes from various marine samples and, in this report, we analyzed marine-derived actinomycetes for acetylcholinesterase inhibitors of the enzyme potential by thin layer chromatography.

An acetylcholinesterase inhibitor or anti-cholinesterase is a chemical that inhibits the cholinesterase enzyme from breaking down acetylcholine so increasing both the level and duration of action of the neurotransmitter acetylcholine. Acetylcholinesterase is a tetrameric protein which catalyzes the hydrolysis of acetylcholine. The active site of AChEase includes a serine hydroxyl group, which is rendered more nucleophilic through the proton-acceptor action of a nearby histidine residue. The serine residue exerts a nucleophilic attack on the carbonyl carbon of ACh. A tetrahedral transition state is reached, which results in serine acetylation and the loss of free choline. The acetyl group binds to histidine as an N-acetate, but is hydrolyzed rapidly to yield free choline, acetate, and the free enzyme.

Acetylcholinesterase inhibitors occur naturally as venoms and poisons are used as weapons in the form of nerve agents are used medicinally to treatment of neurological disorders such as Alzheimer's disease, senile dementia, ataxia and myasthenia gravis.

\section{Materials and Methods}

Cultivation and crude extract preparation. Some strains of isolated actinomycetes will be grown onto Nutrient Agar (NA) medium consisted of nutrient broth, agar and sea water for 3 days at room temperatures. Colony that growing be cut in small pieces of agar block then transferred to each four cultivated media (media A consisted of sediment, chitineous, sea weed and sea water; media B consisted of glycerol 100\%, bacto soytone and sea water; media $\mathrm{C}$ consisted of bio-peptone/polypeptone, yeast extract, soluble starch and sea water; media D consisted of peptone, yeast extract and sea water). Those media will be in shaker incubator for 3 days in $25-30^{\circ} \mathrm{C}$ at $200 \mathrm{rpm} .5 \% \mathrm{v} / \mathrm{v}$ from inoculum will be inoculated again in $100 \mathrm{ml}$ to each four cultivation media for 5- 7 days in $25-30^{\circ} \mathrm{C}$ at $200 \mathrm{rpm}$. 
Actinomycetes Extraction. After 5-7 days of cultivation at $25-30^{\circ} \mathrm{C}, 200 \mathrm{rpm}$, the media include inoculum transferred to separatory funnel and add ethyl acetate with $1: 1$. This work done by three times. Ethyl acetate collected then removed by rotary evaporator yielding the crude extract of actinomycetes and the crude extract stored at $-20^{\circ} \mathrm{C}$ for further screening.

Screening AChE inhibitors of crude extract. Crude extract found from extraction of marine actinomycetes dissolved either in ethyl acetate or in methanol according to their solubility. The crude extract of actinomycetes samples were spotted using a micro haematocrit tube at different points at the lower limit of the activated TLC plate. Previous findings of the solubility test showed that di-cloro methane (DCM) and chloroform have the ability to be the second solvent. In this study, we used 2 chamber glasses each containing methanol/ chloroform (20:80) and methanol/ DCM (10:90) solutions. The TLC plate was then inserted into the chamber glass, solution moves along the plate, and the plate was pulled away from the chamber when the solution reached the upper limit of the tip of the plate. Observation of the elution results of the sample was performed using UV light at wavelengths of $254 \mathrm{~nm}$ and $330 \mathrm{~nm}$. The dots that have been shown, marked with a pencil. Thus, the TLC plate is sprayed with specific reagents (ninhydrin, alkaloids, vanillin, and phosphomolybic acid, or PMA). After spraying the reagent, the TLC plate was dried at a high temperature.

\section{Results and Discussions}

The effect of the addition of polar chloroform solvent was very strong to adjust the polarity of the solvent mixture against certain crude extract actinomycetes (CNA 147, 153, 156,161 , and 162) with appropriate reagents. Whereas the use of DCM polar solvent is very effective to shift polarity only to CNA 148 as seen in Figure 1.

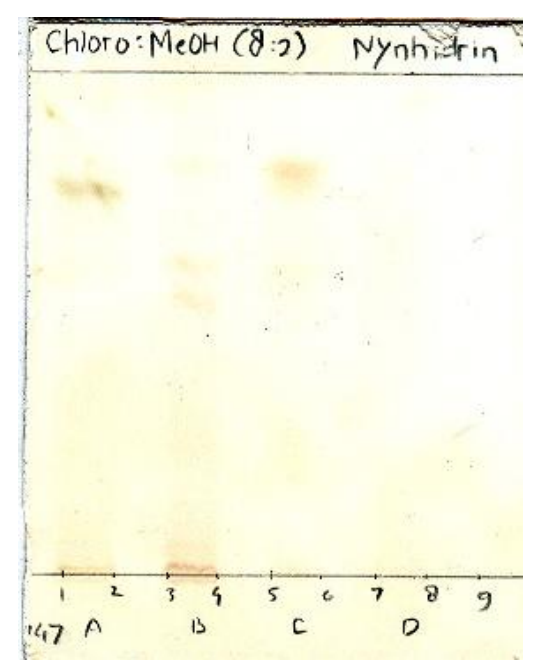

Figure 1. Diagrammatic chromatogram crude extract of Actinomycetes sample CNA 147 with ninhydrin reagent using methanol:chloroform (20:80) solvents

CNA 147 samples sprayed with ninhydrin reagent showed light brown spots (Media A and $\mathrm{C}$ ), while the use of ninhydrin reagent on the sample revealed reddish purple spots. If the sample shows the correct direction of color, it is likely that the sample contains glycoside compounds as one of the phytoconstituent inhibitors of AChE (Figure 1). 
Samples of CNA 153, 156, 161 and 162 with alkaloid reagent spray revealed those samples containing alkaloid compounds. Component of alkaloid with a dark brown color after spraying of the alkaloid reagent. As we note, alkaloid is one of the AChE reliever phytoconstituents (Figures 2a and b).

A sample of CNA 147 sprayed with a vanillin reagent displayed a bright green-yellow color for the identification of amines and amino acid compounds. These colors are seen in the media B, C, and D. Whereas in the media A be streaked, it may be too concentrated (Figure $3)$.

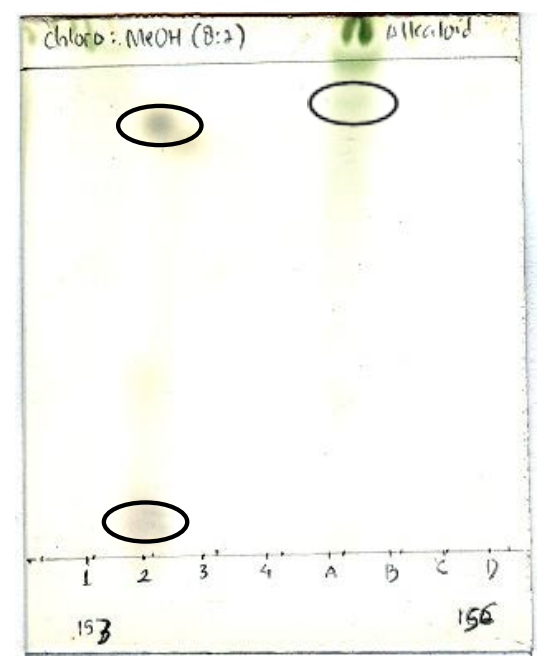

(A)

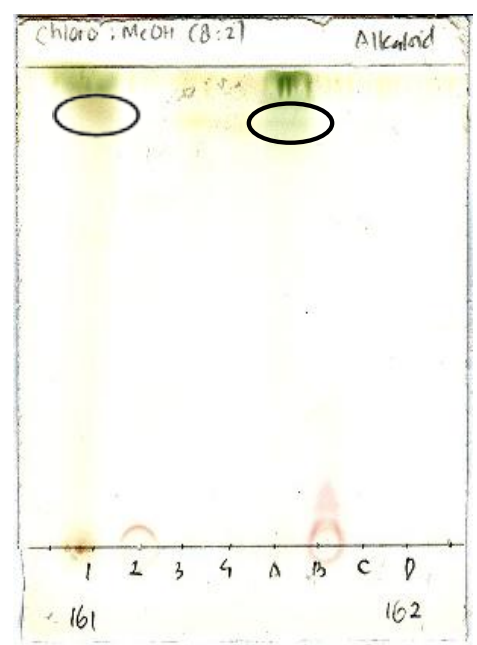

(B)

Figure 2. Diagrammatic chromatogram crude extract of Actinomycetes samples with alkaloid reagent using methanol:chloroform (20:80) solvents. (A) Samples of CNA 153 and CNA 156; (B) Samples of CNA 161 and CNA 162

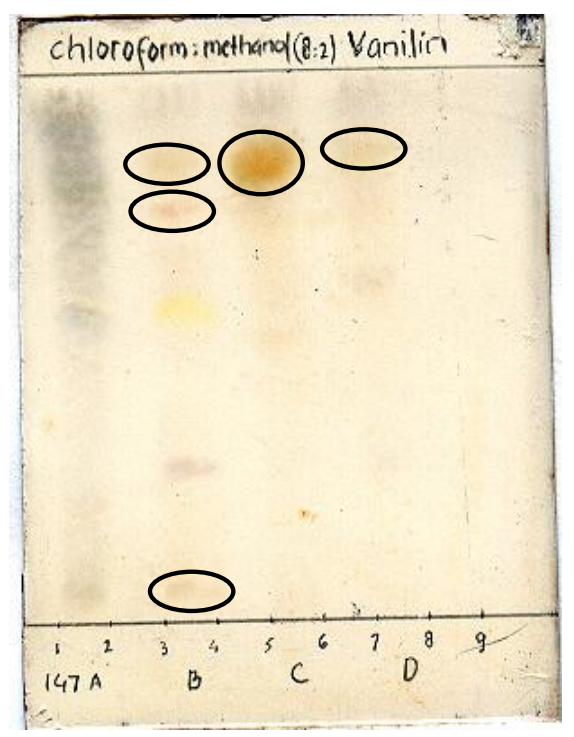

Figure 3. Diagrammatic chromatogram crude extract of Actinomycetes samples of CNA 147 with vannilin reagent using methanol:chloroform (20:80) solvents. 
Phosphomolybic acid reagent used to detect prostaglandins, hormones, lipids, alkaloids and essential oils. The position of the reagent is blue spots when heated at 100 to $110^{\circ} \mathrm{C}$. The CNA 148 sample showed a deep blue-violet, which caused the TLC plate to have overheated. Even so, these spots indicated that the CNA 148 sample contains 2 phytoconstituents of AChE inhibitors (Fig. 4).

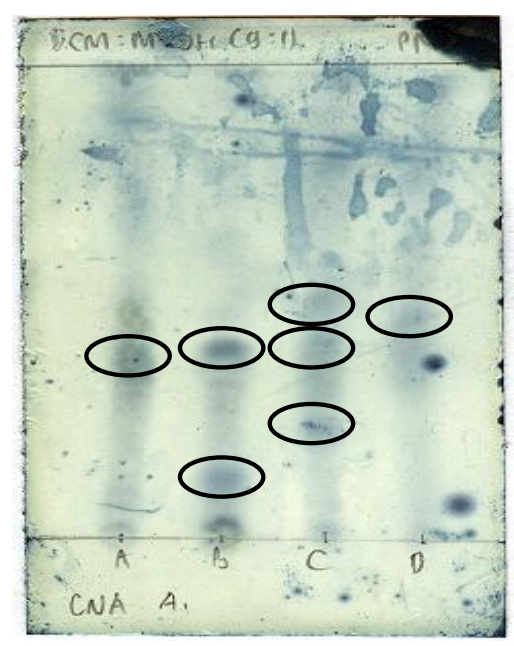

Figure 4. Diagrammatic chromatogram crude extract of Actinomycetes samples of CNA 148 with PMA reagent using methanol:DCM (10:90) solvents.

\section{Conclusion}

The addition of polar solvents (chloroform and DCM) to methanol solvents with unique reagents can detect $\mathrm{AChE}$ inhibitors from crude actinomycetes extracts. The combination of methanol : chloroform $(20: 80)$ resulted in a broader detection of AChE inhibitors than the combination of methanol : DCM (10:90) which only detected 1 sample (CNA 148). 


\section{References}

A.R. de Boer, T. Letzel, D.A. van Elswijk, H. Lingeman, W.M. Niessen, H. Irth, Anal.Chem.76 (2004) 3155.

Camiel F. de Jong, Rico J.E. Derks, Ben Bruyneel, Wilfried Niessen, Hubertus Irth Highperformance liquid chromatography-mass spectrometry-based Acetylcholinesterase assay for the screening of inhibitors in natural extracts. Journal of Chromatography A, 1112 (2006) 303-310

Giovanni, SD., Aline B., Aurelie U., Andrew M., Kurt H., Pierre-Alain C. and Marianne R. In vitro Screening assays to identify natural or synthetic acetylcholinesterase inhibitors: Thin layer chromatography versus microplate methods. Eur J Pharm Sci (2007).

Guardigli, M., Pasini,P., Mirasoli,M., Leoni,A. etal., Analytic Chim. Acta. 2005, $535,139-144$.

Ingkaninan K., Prapapan T., Kanchanaporn C., Thitaree Y., Warawit T., 2003. Screening for acetylcholinesterase inhibitory activity in plants used In Thai traditional rejuvenating and neurotonic remedies. Journal of Ethnopharmacology 89 (2003) 261-264

Ingkaninan, K., de Best, C.M., Irth, H., vander Heijden, R., Hofte, A.J.P., Karabatak, B., Tjaden, U.R., vander Greef, J., Verpoorte, R. 2000. High performance liquid chromatography with on-line coupled UV-mass spectrophotometric-biochemical detection for identification of acetylcholinesterase inhibitors from natural products. Journal of Chromatography A 872, 61-73.

Kuno, F.; K. Shiomi, K. Otoguro, T. Sunazuka \& S. Omura: Arisugacins A and B, novel and selective acetylcholinesterase inhibitors from Penicillium sp. FO-4259. II. Screening, Taxonomy, Fermentation, Isolation and Biological Activity. J. Antibiotics 49: 748-751, 1996

Okabe, H.; K. Sagesaka, A. Noma \& N. Nakajima: Enzymatic assay of cholinesterase activity. II. Clinical application. Jap. J. Clin. Path. 25: 755-758, 1977.

Rhee,I.K., vander Meent, M., Ingkaninan, K., Verpoorte, R., 2001. Screening for acetylcholinesterase inhibitors from Amaryllidaceae using silica gel thin-layer chromatography in combination with bioactivity staining. Journal of Chromatography A 915, 217-223

Robert M. Cohn and Karl S. Roth. 1996. Biochemistry and Disease, Bridging basic science and Clinical practise, USA.

Seung-Kyoung Jeung, Hyun Jae Yim, Hye Mi Kim, Se Gye Shin, Seoung Jun Han, Jung HY Park and Il-Jun Kang. Isolation of Acetylcholinesterase Inhibitors from the Flowers of Chrysanthemum indicum Linne. The FASEB Journal. 2007;21:1b189

Stahl,E., 1961. Duennschicht chromatographieein Laboratoriumshand-buch. Springer-Verlag, Berlin, pp.86-90. 
Susana López, Jaume Bastida, Francesc Viladomat and Carles Codina Acetylcholinesterase inhibitory activity of some Amaryllidaceae alkaloids and Narcissus extracts. Life Sciences Volume 71, Issue 21, 11 October 2002, Pages 2521-2529

Tabet N,. Acetylcholinesterase inhibitors for Alzheimer's disease: anti-inflammatories in acetylcholine clothing! Age and Ageing 2006; 35: 336-338.

Young-Won Chin, Marcy J., Balunas, Hee Byung Chai and A. Douglas Kinghorn. Drug Discovery From Natural Sources . The AAPS Journal 2006; 8 (2) Article 28

Zhang HY, Tang XC. Huperzine B. a novel acetylcholinesterase inhibitor, attenuates hydrogen peroxide induced injury in PC12 cell. Neurosci Lett 2000; 292: 41-4.

Zhong-mei Tang, Zhao-yanWang and Jing-wuKang. $2006 . \quad$ Screening of acetylcholinesterase inhibitors in natural extracts by CE with electrophoretically mediated microanalysis technique. Electrophoresis 2007,28,360-365. 